Address for correspondence: Robin Kobbe, Bernhard Nocht Institute for Tropical Medicine, Infectious Epidemiology, Bernhard-NochtStr. 74, D-20359 Hamburg, Germany, email: kobbe@bni.uni-hamburg.de

\section{Early Diagnosis of Disseminated Mycobacterium genavense Infection}

To the Editor: Nontuberculous mycobacteria are environmental organisms that cause life-threatening diseases, particularly in immunocompromised hosts. They are increasingly recognized for causing problems in the management of solid-organ transplant recipients, due to improved diagnostic methods as well as increasing numbers and life expectancy of these patients (1). The slow-growing $\mathrm{Myco-}$ bacterium genavense is a ubiquitous nontuberculous mycobacterium; it is reportedly isolated from tap water, pets, and the gastrointestinal tract of healthy humans $(1,2)$. It was first recognized as a human pathogen in a patient with AIDS but has not yet been found in heart transplant recipients (3). We report early diagnosis of disseminated $M$. genavense infection in a heart transplant recipient.

A 37-year-old man was hospitalized in September 2001 for abdominal pain, sweats, and weight loss; he had received a heart transplant 3 years earlier. Immunosuppressive treatment, which began immediately after transplantation, consisted of tacrolimus (5 $\mathrm{mg}$ ) and mycophenolate mofetil (2 g) daily; concurrent steroid therapy was tapered off over the next 6 months. A computed tomographic (CT) scan showed numerous large lymph nodes in his abdomen (Figure). Endoscopic examinations showed diffuse inflam- mation of the mucosa of the duodenum, ileum, and colon. Multiple biopsy samples were submitted for histologic analysis, culture, and molecular biological analysis. Immediate $16 \mathrm{~S}$ rRNA gene amplification that used universal primers (4) and sequencing of samples taken directly from the biopsy material led to the identification of $M$. genavense. A 475-bp fragment was sequenced, and $99 \%$ homology with the gene of type strain ATCC 51234 (GenBank accession no. X60070) was found. PCR results were positive for 2 of the 4 samples tested. The molecular identification was compatible with the subsequent histologic finding of profound macrophage infiltration without granuloma and the presence of Ziehl-Neelsen-positive bacilli. Five weeks later, the molecular diagnosis was confirmed by blood cultures and cultures of the intestinal mucosa samples (Inno-LiPA Mycobacteria test, version 2, Innogenetics, Courtaboeuf, France). The direct molecular diagnosis of $M$. genavense enabled immediate treatment of the patient with the combination of moxifloxacin, ethambutol, clarithromycin, and amikacin; mycophenolate mofetil was discontinued. Clofazimine was added to the treatment regimen 3 months later, when a control CT scan showed that some of the enlarged mesenteric lymph nodes had increased further. After 5 months, the clinical signs resolved, and after 9 months, the lymph nodes were substantially smaller. CT scan results were within normal limits after 12 months of treatment; only ethambutol and clarithromycin were continued for an additional 6 months. There was no sign of $M$. genavense infection relapse 3 years after the diagnosis had been made.

Nontuberculous mycobacteria in persons who have received heart or other solid-organ transplants remain a rare cause of late infectious complications and occur $\approx 3.5$ years after transplantation $(1,4)$. In the subgroup of heart transplant recipients, skin disease is the most common clinical manifestation, followed by pulmonary and disseminated disease; $M$. kansasii, $M$. avium complex, and $M$. haemophilum infections are most frequently encountered $(1,5)$.

M. genavense causes up to $12.8 \%$ of all nontuberculous mycobacteria infections in AIDS patients; these infections are clinically similar to those caused by the M. avium complex $(1,6)$.

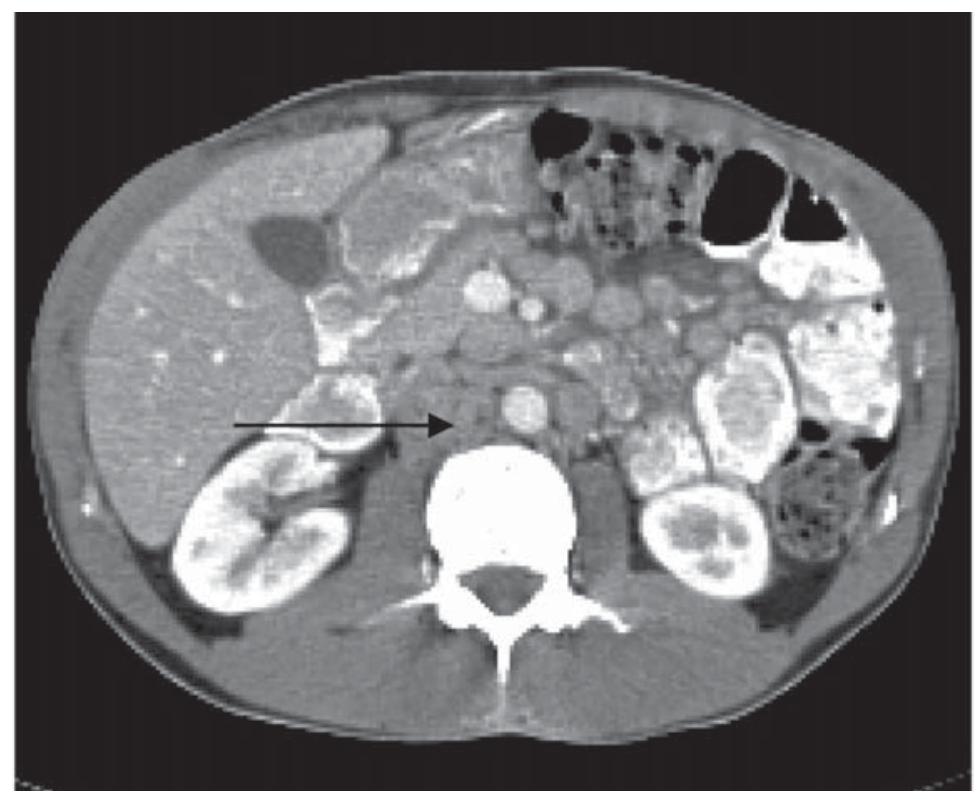

Figure. Initial computed tomographic scan of the abdomen, performed after intravenous injection of contrast dye, showing numerous enlarged para-aortic lymph nodes (arrow). 
M. genavense infections occur only rarely in persons other than AIDS patients (as in the present case), but they always occur in immunocompromised persons $(7,8)$. To date, only 1 case of disseminated infection has been reported in a solid-organ (kidney) transplant recipient; the diagnosis was made by molecular identification in isolates from blood and marrow cultures. That patient died of complications from $M$. genavense infection (9). Because $M$. genavense is a fastidious organism, the infections it causes are difficult to diagnose and their frequency is probably underestimated, which may change with increased use of direct molecular biological methods.

Optimal treatment of $M . g e-$ navense infections has not been established (10). Experience with $M$. genavense infections in AIDS patients and with other nontuberculous mycobacteria infections in solid-organ transplant recipients suggests that at least 2 antimicrobial drugs should be used for a prolonged period; when possible, immunosuppressive drugs should be concurrently reduced $(1,3,6,10)$. Outcome of nontuberculous mycobacteria infections in transplant patients is highly variable $(1,5)$ but was satisfactory in the present patient, who was treated with quintuple antimicrobialdrug therapy and reduced immunosuppressive therapy.

This case of a disseminated infection due to $M$. genavense in a heart transplant recipient was diagnosed early. Universal $16 \mathrm{~S}$ rRNA gene sequencing after amplification directly from intestinal biopsy specimens enabled fast diagnosis and appropriate management.

We gratefully acknowledge a grant (to I.P.) from Assistance Publique-Hôpitaux de Paris (Innovations et Centre d'Investigation Biomédicale: Transfert de technologies en biologie ou en imagerie).

\section{Victoire de Lastours, ${ }^{*} \dagger$ Romain Guillemain, ${ }^{*} \dagger$ Jean-Luc Mainardi, ${ }^{*}+£$ Agnès Aubert, ${ }^{*} \dagger$ Patrick Chevalier, ${ }^{*} \dagger$ Agnès Lefort, *ף and Isabelle Podglajen, ${ }^{*} † \S$}

*Assistance Publique-Hôpitaux de Paris, Paris, France; †Hôpital Européen Georges Pompidou, Paris, France; łUniversité Paris-Descartes, Paris, France; §Université Pierre et Marie Curie, Paris, France; and đHôpital Beaujon, Paris, France

\section{References}

1. Doucette K, Fishman JA. Nontuberculous mycobacterial infection in hematopoietic stem cell and solid organ transplant recipients. Clin Infect Dis. 2004;38:1428-39.

2. Dumonceau JM, Fonteyne PA, Realini L, Van Gossum A, Van Vooren JP, Portaels F. Species-specific Mycobacterium genavense DNA in intestinal tissues of individuals not infected with human immunodeficiency virus. J Clin Microbiol. 1995;33:2514-5.

3. Bottger EC, Teske A, Kirschner P, Bost $\mathrm{S}$, Chang HR, Beer V, et al. Disseminated "Mycobacterium genavense" infection in patients with AIDS. Lancet. 1992;340:7680.

4. Relman DA, Schmidt TM, MacDermott RP, Falkow S. Identification of the uncultured bacillus of Whipple's disease. N Engl J Med. 1992;327:293-301.

5. Novick RJ, Moreno-Cabral CE, Stinson EB, Oyer PE, Starnes VA, Hunt SA, et al. Nontuberculous mycobacterial infections in heart transplant recipients: a seventeen-year experience. J Heart Transplant. 1990;9:357-63.

6. Pechère $\mathrm{M}$, Opravil $\mathrm{M}$, Wald $\mathrm{A}$, Chave JP, Bessesen M, Sievers A, et al. Clinical and epidemiologic features of infection with Mycobacterium genavense. Arch Intern Med. 1995;155:400-4.

7. Krebs T, Zimmerli S, Bodmer T, Lammle B. Mycobacterium genavense infection in a patient with long-standing chronic lymphocytic leukaemia. J Intern Med. 2000;248:343-8.

8. Bogdan C, Kern P, Richter E, Tannapfel A, Rüsch-Gerdes S, Kirchner T, et al. Systemic infection with Mycobacterium genavense following immunosuppressive therapy in a patient who was seronegative for human immunodeficiency virus. Clin Infect Dis. 1997;24:1245-7.
9. Nurmohamed S, Weenink A, Moeniralam $\mathrm{H}$, Visser C, Bemelman F. Hyperammonemia in generalized Mycobacterium genavense infection after renal transplantation. Am J Transplant. 2007;7:722-3.

10. Albrecht H, Rusch-Gerdes S, Stellbrink HJ, Greten H. Treatment of disseminated Mycobacterium genavense infection. AIDS. 1995;9:659-60.

Address for correspondence: Isabelle Podglajen, Assistance Publique-Hôpitaux de Paris, Hôpital Européen Georges Pompidou, Service de Microbiologie, 20 rue Leblanc, 75908 Paris CEDEX 15, France; email: isabelle.podglajen@ hop.egp.ap-hop-paris.fr

Isolation of Novel Adenovirus from Fruit Bat (Pteropus dasymallus yayeyamae)

To the Editor: Bats are thought to be one of the most important reservoirs for viruses such as Nipah virus, severe acute respiratory syndrome (SARS) coronavirus, and Ebola virus (1). These pathogens became known after extensive surveys of bats following outbreaks. As a first step in investigating unidentified pathogens in bats and to help forecast the potential threat of emerging infectious diseases, we tried to isolate and characterize viruses that persistently infect bats. In the process, we isolated a novel adenovirus from a fruit bat in Japan.

Pteropus dasymallus yayeyamae, or Ryukyu flying fox, is a fruit bat of Japan. With the permission of the governor of Okinawa, we caught 1 adult male bat of this species and used its spleen and kidneys to establish primary cell cultures. On the 4th passage of the primary adherent cells derived from the spleen, a cytopathic effect (CPE) appeared without any visible 